\title{
Understanding the Impact of Using Ontology Matching Tools for Validating Feature Models with Domain Knowledge
}

\author{
Nada M. Hassan \\ Arab Academy for Science, Technology \\ and Maritime Transport \\ Cairo, Egypt \\ nadamahmoudhassan@aast.edu
}

\author{
Haitham S. Hamza Member, IEEE \\ Cairo University \\ Giza, Egypt \\ hhamza@fci-cu.edu.eg
}

\author{
Yasser M. K. Omar \\ Arab Academy for Science, Technology \\ and Maritime Transport \\ Cairo, Egypt \\ dr_yasser_omar@yahoo.com
}

\begin{abstract}
${ }^{1}$ Abstract - Feature models (FM) are a way for modeling and describing the product family of specific domain. They are widely used for describing the requirements in the domain engineering as it describes the commonalities and the differences of related products in specific domain. Currently the research in the feature model analysis and the validation of it focus on capturing the inconsistencies of the feature configurations of software systems. However the semantic web had been used for representing the feature models as ontology using OWL DL to use the Description Logic (DL) reasoners in validating the consistency of the feature model configurations, detecting the semantic contradictions or semantic mappings with certain domain is missed. The aim of this research is to detect the semantic mappings between a feature model and a specific domain ontology using the ontology marching tools. In the paper we used the Wireless Sensor Actuator Network (WSAN) feature model for analysis, and the well-known Semantic Sensor Network ontology (SSN) for validation. Two ontology-matching tools are used to map the feature model and the domain ontology, and the results have been compared.
\end{abstract}

Keywords—feature model; ontology; description logic; ontology matching; semantic mapping; wireless sensor network

\section{INTRODUCTION}

Software product lines (SPLS) depend on the idea of producing a family of software products from common features instead of producing them from scratch. The feature model has been firstly introduced in the Feature-Oriented domain analysis (FODA) method as a structure handles the product line members and determining the features that differentiate the software systems in a specific domain [1].

Feature models are most widely used for managing commonalities and variabilities of the features between different systems in a certain domain.

Introducing a new member to SPL, requires feature configurations to define the set of features that describe this member and this configurations are accepted if it doesn't violate the constraints defined by the feature model.The automated analysis of FMs is used to check the validity of the combination of features in a product. This process is mainly

DOI reference number: 10.18293/SEKE2017-199 carried out in two-phases. "In the first phase, the feature model is converted into a specific representation (e.g. Propositional Logic, Description Logic, etc). Then in the second phase, an off-the-shelf solver or some algorithms are used to analyze the representation of the feature model parameters automatically and provide the result" [2]. Validating the feature model configurations and consistency doesn't reveal the semantic contradictions or semantic mappings with certain domain. This may lead to generate product with illogic features with respect to its domain.

To the best of our Knowledge, none of the existing studies that used the power of DL focused on validating the feature model with certain domain. Detecting the semantic mappings between a feature model and a certain domain may help in the process of validating a feature model with domain knowledge.

In this paper we present how we can use the ontology matching tools to detect the semantic mappings between WSAN FM and SSN ontology. We used the OWL DL to represent the FM, then we tested two ontology matching tools. The paper is organized as follows: sectionII presents the background. Section III provides the related work. Section IV presents a proposed hybrid model applied on a feature model for WSAN [5]. Section V demonstrates the Experimental Results followed by the Conclusion and Future work.

\section{BACKGROUND}

This section explains the basis of the feature models and the ontology matching techniques.

\section{A. Feature Models}

Feature models are used for representing the features of SPL. They have a tree structure. The model starts with root feature followed by sub-features with different relationships with its parent feature. The main types of the FM relations are:

- Mandatory: means the feature must be included into the description of a concept

- Optional: means the existence of the feature is optional

- Alternative: means just one feature from a set of features can be included

- OR: means one feature or more can be included from a set of features. 


\section{B. Ontology Matching Techniques}

Ontology matching is a complicated process that helps in detecting the semantic correspondences between different ontologies of the same domain, the matching techniques are divided mainly into two types:

\section{- $\quad$ Element-Level technique}

This technique is based on obtaining the correspondences between the entities with ignoring the structure of the ontology, this technique is divided into string-based, language-based and linguistic resource as in [14].

\section{- $\quad$ Structure-Level technique}

This technique focuses on obtaining the correspondences based on the relations between the entities within the structure of the ontology, this technique is divided into taxonomy mapping, and tree-based mapping as in [14].

\section{RELATED WORK}

This Section is divided into two parts: Related work of the automated analysis of the Feature Models using DL and representing Feature Model in OWL.

\section{A. Automated Analysis of Feature Models}

Wang, Hai H., Yuan Fang Li, Jing Sun, Hongyu Zhang, and Jeff Pan were the first to use the DL in feature model analysis to check the feature model consistency with specific configurations. They represent the FM as ontology using OWL DL, then they used DL Racer and FACT++ to check the consistency of the feature configurations automatically, and also they proposed a debugging OWL tool[6].

Zaid, Lamia Abo, Frederic Kleinermann, and Olga De Troyer proposed an ontology framework for feature modeling that consists of an ontology that formally provides the structure of feature models to check the consistency of FM through rule based model, and provide means to integrate segmented feature models[7].

\section{B. Representing Feature Model in OWL}

As mentioned above, different ways for representing the feature model as ontology had been introduced in [6] and [7].

Tenório, Thyago, Diego Dermeval, and Ig Ibert Bittencourt proposed a generic ontology called OntoSPL represents the structure of the feature model and its relations using OWL classes and properties, so the features can be represented as OWL individuals from this structure, this approach is useful for the dynamic changes at the runtime, although it's not tested on large feature model[8].

Nima Kaviani, Bardia Mohabbati, Dragan Gasevic, and Matthias Finke used the approach that proposed by Wang et al to represent the feature model in ontology to cover non functional requirements, annotate and expand feature models context of ubiquitous environments[9].

As the approach in [6] has been used in other research work, and they tested their approach on a feature model for a large system which contains almost 1000 different features and more than 400 different feature relations, then they applied a debugging process on it. So it is decided to use this approach in the step of representing the feature model in OWL.

\section{PROPOSED HYBIRD MODEL}

Our experimental work tested on the WSAN feature model and the SSN ontology, we used two ontology matching tools to detect the mappings between them, Fig. 1 shows the steps of our experimental work.

WSAN is composed of large number of sensor nodes and actuators to sense, actuate and communicate to provides specific functionality, While the SSN ontology describes sensors and its properties, capabilities and observations[11].

The experimental work divided into two main parts:

\section{A. Constructing the ontology from WSAN}

We used the approach in [6], which presents how to use the OWL DL to represent the FM. They define OWL class for each feature in the FM and make them mutually disjoint then using existential restrictions they bind the rule classes that has been created for each feature to the corresponding produced OWL class, each rule class is used to define the constraints over the feature (OWL class).

Table I. contains the DL syntax that used in representing the axioms of the produced ontology (WSAN ontology)

It can be seen from Fig. 2 that the first level of the WSAN FM contains mandatory feature (i.e. Communication) that can be represented in DL as shown below.

Communication $\sqsubseteq T$ CommunicationRule $\sqsubseteq T$

hasCommunication $\sqsubseteq$ ObjectProperty

$T \sqsubseteq$ hasCommunication.Communication

CommunicationRule $\equiv \exists$ hasCommunication.Communication

WSAN $\sqsubseteq \exists$ hasCommunication.Communication

TABLE I. SUMMERY TABLE OF THE DL SYNTEX USED IN THIS PAPER

\begin{tabular}{|l|l|}
\hline Notation & Explanation \\
\hline$T$ & Superclass of all OWL classes \\
\hline A $\subseteq$ B & A is a subclass of B \\
\hline A $\sqcup$ B & Class union \\
\hline A $\equiv$ B & Class equivalence \\
\hline$\exists=\forall$ P.A & $\begin{array}{l}\text { allValuesFrom =someValuesFrom restriction for every } \\
\text { instance of this class that has instances of property P, all } \\
\text { some of the values of the property are members of class A }\end{array}$ \\
\hline
\end{tabular}

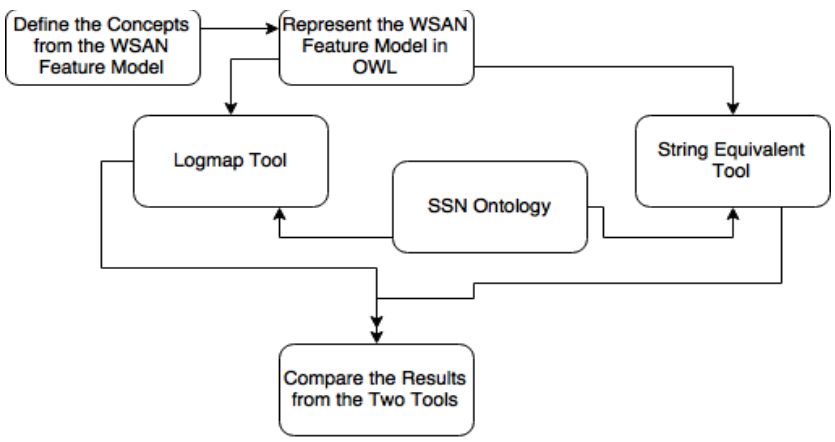

Fig. 1. Block diagram for the steps of the proposed model 


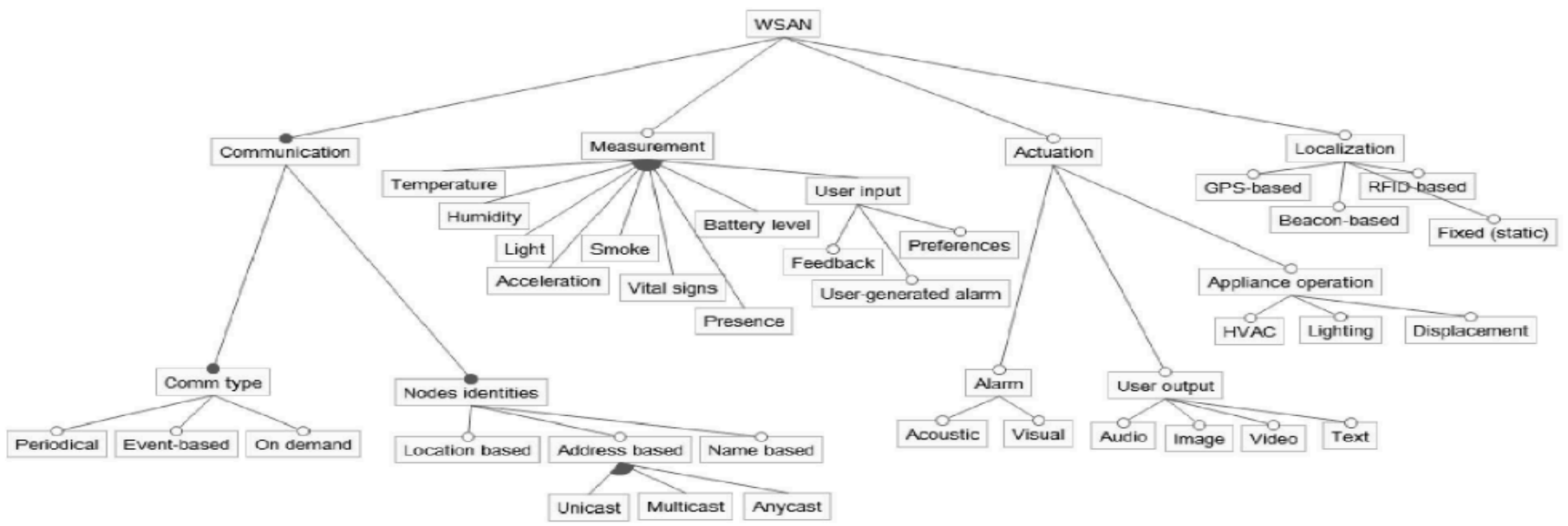

Fig. 2. Feature Model of Wireless Sensor Actuator Network [5]

In Fig.2 the optional features of the first level (e.g. Measurement, etc...) can be represented in DL as shown.

Measurement $\sqsubseteq T$ MeasurementRule $\sqsubseteq T$

hasMeasurement $\sqsubseteq$ ObjectProperty

MeasurementRule $\equiv \exists$ hasMeasurement.Measurement

The Address-based feature has three sub-features with OR relation as shown in Fig.2 that can be represented in DL as shown assuming the Rule classes have been created for each.

AddressBasedRule $\sqsubseteq((\exists$ hasUnicast.Unicast $) \sqcup(\exists$ hasMulticast.Multicast $) \sqcup$ ( $\exists$ hasAnycast.Anycast $))$

The rest of the features of the WSAN feature model have been represented in the same way .

\section{B. Define the mappings between the WSAN produced ontology and SSN ontology}

The produced ontology from WSAN FM is used to be mapped with SSN ontology using the ontology matching tools. We used two ontology matching tools (i.e. Logmap, String Equality tool) to generate the mappings.

\section{- Log-Map}

The LogMap tool is developed by the University of Oxford. The tool depends on lexically and structurally indexing the input ontologies as the matching process is carried out through five phases[12].

\section{A. Lexical indexation}

Logmap identifies for each input ontology the classes labels and its lexical variants using external lexicons (i.e. WordNet, UMLS lexicon).The UMLS lexicon is a set of files that contains many biomedical terminologies, and vocabularies.

\section{B. Structural indexation}

The logmap creates the extended class hierarchy through the use of structural heuristics or DL reasoner for the input ontologies.

\section{Computation of initial (anchor mappings)}

Logmap computes the exact lexical correspondences which are act as initial set of anchor mappings by intersecting the lexical indexes of each input ontology.

\section{Mapping repair and discovery}

- In the repair process, the Log-Map detects the unsatisfiable classes from merging the two input ontologies using a reasoning algorithm, and then it repairs these undesirable correspondences using a greedy diagnosis algorithm.

- For the discovery phase, according to two sets of semantically related classes new mappings are computed by matching the classes in these two sets using a tool that computes a similarity score.

\section{E. Ontology overlapping estimation}

Logmap creates two modules for each ontology, these modules represent the overlapping between $\mathrm{O} 1$ and $\mathrm{O} 2$. If a correct mapping between $\mathrm{O} 1$ and $\mathrm{O} 2$ is missed by logmap, when manually looking in these modules you can find it.

\section{- $\quad$ String Equality tool}

The String equality tool had been proposed in [10]. This tool is based on string based technique and semantic technique. It works to find the similar classes between input ontology files. The algorithm is based on using synonym file that contains the terms and synonyms of the domain that the input ontologies related to it and also detect the exact mappings.

Finally, the results of the ontology matching tools (i.e. Logmap tool, String Equality tool) were compared and evaluated according to predefined expected results.

\section{EXPERIMENTAL RESULTS}

We tested two ontology matching tools (i.e. Logmap tool and String equality tool) to map WSAN ontology and the SSN ontology. The expected results from the matching process are shown in TableII, while the comparison between the two tools and its output are shown in TableIII. Followed by Fig3. that shows part of the SSN ontology.

TableIII. shows no classes mapped using logmap, although it generates one object property mapping. The object property "hasLocation" in SSN mapped with "hasLocalization" object property in WSAN but semantically both of these relations connect classes with different semantic meaning and represent different context, also it mapped wrongly the "Measurement capability" in SSN with "Measaurement" in WSAN and both of them describe different context. 
TABLE II. EXPECTED RESULTS

\begin{tabular}{|c|l|l|}
\hline Ontology & WSAN & SSN \\
\hline \multirow{3}{*}{ Mapped Classes } & WSAN & System \\
\cline { 2 - 3 } & Communication_Type & Stimulus \\
\cline { 2 - 3 } & Communication_Type & Sensor_Input \\
\hline
\end{tabular}

TABLE III. COMPARISON BETWEEN THE TESTED ONTOLOGY MATCHING TOOLS

\begin{tabular}{|c|c|c|c|}
\hline Tool & Input & Output & $\begin{array}{c}\text { Ontology Matching } \\
\text { Technique }\end{array}$ \\
\hline Log-Map & $\begin{array}{l}\text { File1: WSAN } \\
\text { Ontology } \\
\text { File2: SSN } \\
\text { Ontology }\end{array}$ & $\begin{array}{l}\text { (1) Class } \\
\text { mapped } \\
\text { (1) Object } \\
\text { Property } \\
\text { mapped }\end{array}$ & $\begin{array}{l}\text { Based on obtaining } \\
\text { initial set of mappings, } \\
\text { then it use these } \\
\text { mappings to discover } \\
\text { new mappings }\end{array}$ \\
\hline $\begin{array}{c}\text { String } \\
\text { Equality } \\
\text { Tool }\end{array}$ & $\begin{array}{l}\text { File1: WSAN } \\
\text { Ontology } \\
\text { File2: SSN } \\
\text { Ontology }\end{array}$ & $\begin{array}{c}\text { (2) Class in } \\
\text { WSAN mapped } \\
\text { with (3) classes } \\
\text { in SSN }\end{array}$ & $\begin{array}{l}\text { Based on string based } \\
\text { technique, language } \\
\text { based method and } \\
\text { semantic technique, it } \\
\text { uses external file } \\
\text { contains the synonyms } \\
\text { of the domain }\end{array}$ \\
\hline
\end{tabular}

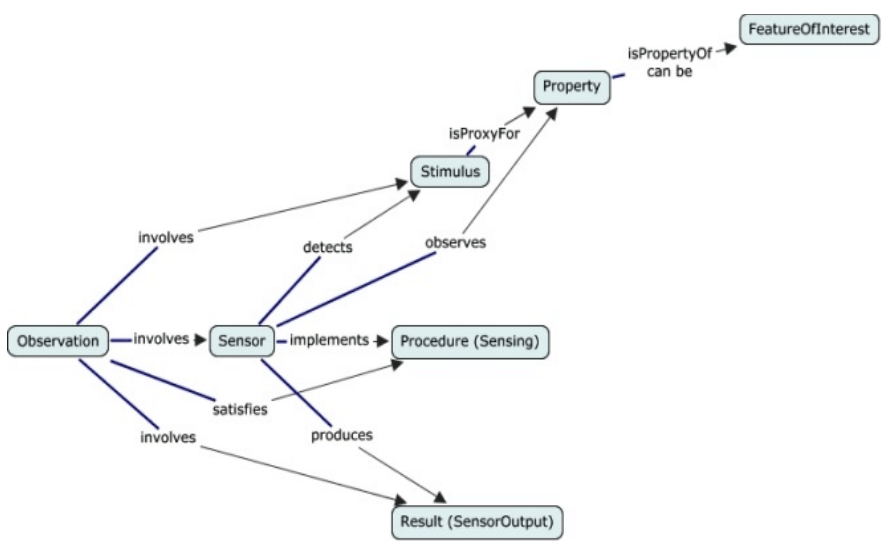

Fig. 2. Part of the Semantic Sensor Network [13]

On the other hand the String equality tool gets two mappings between the input ontologies. The "communicationType" class in WSAN ontology which represents the stimulus that triggers the sensors to work is mapped with "Stimulus" class in Fig.3 and "Sensor_Input" class that are equivelent classes in SSN ontology which represent the stimulus that triggers the sensor, and also it mapped the "WSAN" with "System" in SSN.

According to the expected results in TableII and the output in TableIII, the String Equality tool shows better results than the Logmap tool. LogMap doesn't produce all the mappings in the nonbiomedical domains.

The results of the mapping can be considered as a resonable results, as the SSN ontology describes the sensors and its observations, while the WSAN describes the Network of connected wireless sensors and actuators.

\section{CONCLUSION AND FUTURE WORK}

This paper presented a comparison between two different matching tools (i.e. LogMap tool, String Equality tool) that have been used for detecting the semantic mappings between the WSAN FM and SSN ontology as a domain knowledge using the ontology matching tools. The OWL DL is used for representing the FM as ontology using wang et al approach.

We can conclude from the results that the generated mappings can help in determining to what extent a feature model matches a certain domain, as in our experiement the WSAN FM wasn't completely matched with the SSN Ontology.The results also shows, that the String Equality tool shows better results than the logmap in our experiment. Therefore having lexicon for the terminologies of the domain that the maping is done over it, gives better results.

In the future work, it's intended to extend this work and test it on other domains to complete the process of the validation and draw recommendations for the features that can be added to the feature model from the ontology.

\section{REFERENCES}

[1] K. Kang, S. Cohen, J. Hess, W. Novak, and S. Peterson, "FeatureOriented Domain Analysis (FODA) Feasibility Study". Technical Report CMU/SEI-90-TR-21, Software Engineering Institute, Carnegie Mellon University, November 1990.

[2] Benavides, David, Sergio Segura, and Antonio Ruiz-Cortés. "Automated analysis of feature models 20 years later: A literature review." Information Systems 35, no. 6 (2010), pp. 615-636.

[3] Kim, Chang Hwan Peter. On the relationship between feature models and ontologies. University of Waterloo, 2006.

[4] Otero-Cerdeira, Lorena, Francisco J. Rodríguez-Martínez, and Alma Gómez-Rodríguez. "Ontology matching: A literature review." Expert Systems with Applications 42, no. 2 (2015), pp. 949-971.

[5] Ortiz, Óscar, Ana Belén García, Rafael Capilla, Jan Bosch, and Mike Hinchey. "Runtime variability for dynamic reconfiguration in wireless sensor network product lines." In Proceedings of the 16th International Software Product Line Conference-Volume 2, pp. 143-150. ACM, 2012.

[6] Wang, Hai H., Yuan Fang Li, Jing Sun, Hongyu Zhang, and Jeff Pan. "Verifying feature models using OWL." Web Semantics: Science, Services and Agents on the World Wide Web 5, no. 2 (2007), pp. 117129.

[7] Zaid, Lamia Abo, Frederic Kleinermann, and Olga De Troyer. "Applying semantic web technology to feature modeling." In Proceedings of the 2009 ACM symposium on Applied Computing, ACM 2009, pp. 1252-1256.

[8] Tenório, Thyago, Diego Dermeval, and Ig Ibert Bittencourt. "On the use of ontology for dynamic reconfiguring software product line products." In Proceedings of the ninth international conference on software engineering advances, 2014, pp. 545-550.

[9] Kaviani, Nima, et al. "Semantic annotations of feature models for dynamic product configuration in ubiquitous environments." 4th International Workshop on Semantic Web Enabled Software Engineering. at 7th International Semantic Web Conference. 2008.

[10] Gawich, M., et al. "Alternative Approaches for Ontology Matching." International Journal of Computer Applications 49.18 (2012).

[11] Compton, Michael, Payam Barnaghi, Luis Bermudez, RaúL GarcíACastro, Oscar Corcho, Simon Cox, John Graybeal et al. "The SSN ontology of the W3C semantic sensor network incubator group." Web semantics: science, services and agents on the World Wide Web 17 (2012), pp. 25-32.

[12] Jiménez-Ruiz, Ernesto, and Bernardo Cuenca Grau. "Logmap: Logicbased and scalable ontology matching." International Semantic Web Conference. Springer Berlin Heidelberg, 2011.

[13] Available:https:/www.w3.org/2005/Incubator/ssn/XGR-ssn-20110628/. [Accessed:10-1-2016]

[14] Hooi, Yew Kwang, M. Fadzil Hassan, and Azmi M. Shariff. "A survey on ontology mapping techniques." In Advances in Computer Science and its Applications, pp. 829-836. Springer Berlin Heidelberg, 2014 